\title{
CONTROL OF INTERVAL SYSTEMS BY USING TWO FEEDBACK CONTROLLERS
}

\author{
Radek Matušu \\ Centre for Security, Information and Advanced Technologies (CEBIA - Tech) \\ Faculty of Applied Informatics, Tomas Bata University in Zlín \\ nám. T. G. Masaryka 5555, 76001 Zlín, Czech Republic
}

\begin{abstract}
This contribution is focused on polynomial synthesis of continuous-time control systems with two feedback controllers and especially on its application to interval plants. Robust stability of designed control loops is investigated through the graphical method which combines the value set concept with the zero exclusion condition. A third order interval system is robustly stabilized in the presented simulation example.
\end{abstract}

Keywords: Two feedback controllers; interval systems; polynomial control; Diophantine equations; robust stability analysis
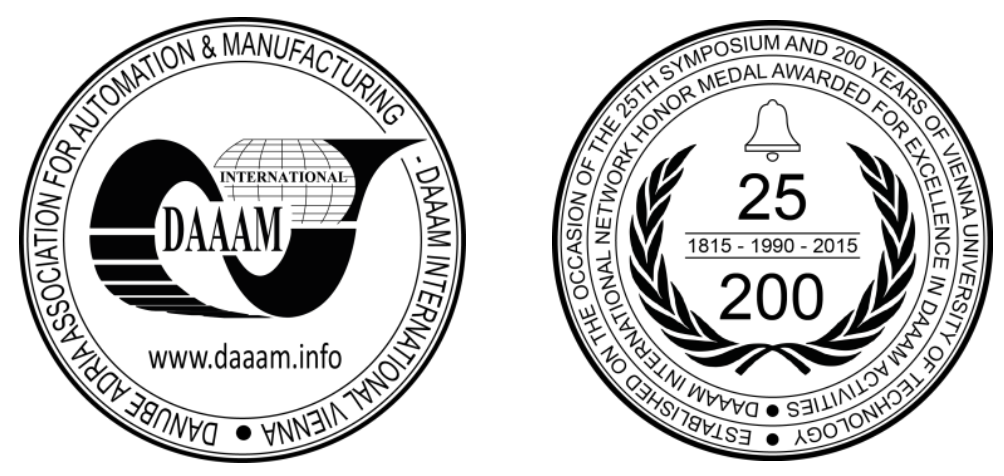

This Publication has to be referred as: Matusu, R[adek] (2016). Control of Interval Systems by Using Two Feedback Controllers, Proceedings of the 26th DAAAM International Symposium, pp.0217-0222, B. Katalinic (Ed.), Published by DAAAM International, ISBN 978-3-902734-07-5, ISSN 1726-9679, Vienna, Austria DOI:10.2507/26th.daaam.proceedings.030 


\section{Introduction}

The control system with two feedback controllers (TFC) is a configuration in which the weight coefficients for two regulators can be chosen [1-4] and thus it offers more tuning facilities in comparison with standard 1DOF or 2DOF [5] control structures. Nevertheless, the robustness of the TFC loops under parametric uncertainty has not been studied in many research works yet.

The main aim of this contribution is to present application of a graphical method of robust stability analysis to continuous-time closed control loops with TFC and interval plants. The applied control synthesis method is based on the polynomial approach [1,2] and solution of Diophantine equations [6]. The following robust stability tests of the resulting closed-loop characteristic polynomials with affine linear uncertainty structure utilize the combination of the value set concept and the zero exclusion principle [7]. In the simulation example, a third order interval plant is robustly stabilized by using TFC structure. The similar ideas as in this work have been already published in the conference paper [3] and its extended version [4].

\section{Synthesis of control system with TFC}

The structure of the control loop with TFC $C_{Q}(s)=\tilde{q}(s) / \tilde{p}(s)$ and $C_{R}(s)=r(s) / \tilde{p}(s)$ and controlled plant $G(s)=b(s) / a(s)$ is shown in Fig. 1. It is adopted from [1,2] with referred original inspiration in [8].

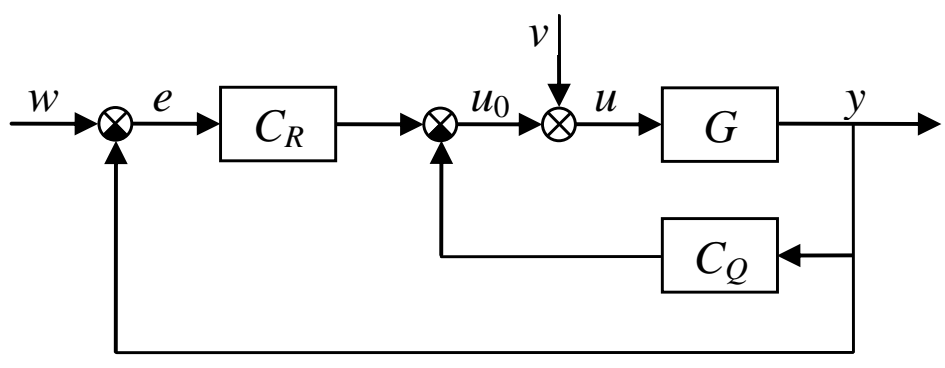

Fig. 1. Control system with TFC

For control design, a polynomial method [1, 2], which helps fulfill the basic requirements such stability and internal properness of the control system, asymptotic tracking of the reference signal and load disturbance rejection, is utilized.

In brief, a fundamental Diophantine equation which is critical for control design is:

$a(s) \tilde{p}(s)+b(s) t(s)=d(s)$

where $t(s)=r(s)+\tilde{q}(s)$. Stability of control loop from Fig. 1 is assured for polynomials $\tilde{p}(s)$ and $t(s)$ obtained as a solution of the equation (1) with a stable right-hand polynomial $d(s)$. In this paper, both reference $w$ and load disturbance $v$ are supposed as stepwise signals, i.e. $W(s)=w_{0} / s, V(s)=v_{0} / s$. Under this presumption, the asymptotic tracking and load disturbance rejection are fulfilled for the polynomials $\tilde{p}(s)=s p(s), \tilde{q}(s)=s q(s)$.

The forms of polynomials $t(s), r(s)$ and $q(s)$ are:

$t(s)=\sum_{i=0}^{n} t_{i} s^{i} ; \quad r(s)=\sum_{i=0}^{n} r_{i} s^{i} ; \quad q(s)=\sum_{i=1}^{n} q_{i} s^{i-1}$

with basic relations among their coefficients [2]:

$r_{0}=t_{0}$
$r_{i}+q_{i}=t_{i} \quad$ for $i=1, \ldots, n$

The coefficients of the polynomials $r(s)$ and $q(s)$ can be obtained on the basis of calculated polynomial $t(s)$ and adjustable coefficients $\gamma_{i} \in\langle 0,1\rangle$ according to:

$r_{i}=\gamma_{i} t_{i} \quad$ for $i=1, \ldots, n$
$q_{i}=\left(1-\gamma_{i}\right) t_{i} \quad$ for $i=1, \ldots, n$ 
Evidently, the coefficients $\gamma_{i}$ represent the weights for numerators of controllers' transfer functions. The unit coefficients $\gamma_{i}$ for all $i$ reduce the control system from Fig. 1 to standard 1DOF configuration $\left(C_{Q}(s)=0\right)$. On the other hand, if $\gamma_{i}=0$ for all $i$ and moreover reference and load disturbance are stepwise signals, the control loop corresponds to $2 \mathrm{DOF}$ structure [2].

However, above all, the control behaviour can be influenced by choice of right-hand polynomial $d(s)$ in Diophantine equation (1). In this paper, just the simplest method with multiple real roots will be employed. The details of the method can be found in [1-4].

\section{Robust stability investigation for systems with parametric uncertainty}

Systems with parametric uncertainty are assumed to have known fixed structure (order) but their real physical parameters are known imprecisely. Usually, such parameters are bounded by intervals with minimal and maximal possible values. Transfer function which describes a plant with parametric uncertainty has a general form:

$$
G(s, q)=\frac{b(s, q)}{a(s, q)}
$$

where $q$ is a vector of real uncertain parameters defined by some uncertainty bounding set (commonly through $L_{\infty}$ norm).

A frequently used representative of systems with parametric uncertainty is the interval plant:

$$
G(s, b, a)=\frac{\sum_{i=0}^{m}\left[b_{i}^{-} ; b_{i}^{+}\right] s^{i}}{\sum_{i=0}^{n}\left[a_{i}^{-} ; a_{i}^{+}\right] s^{i}}
$$

with mutually independent parameters bounded via their lower and upper limits.

From the robust stability analysis viewpoint, the typical object of interest is the uncertain closed-loop characteristic polynomial:

$$
p(s, q)=\sum_{i=0}^{n} \rho_{i}(q) s^{i}
$$

where $\rho_{i}(q)$ are coefficient functions. Then, the related family of closed-loop characteristic polynomials is:

$$
P=\{p(s, q): q \in Q\}
$$

The selection of suitable method for robust stability investigation depends primarily on the uncertainty structure. Mostly, the higher level of relation among coefficients means more complicated testing and thus it usually entails the necessity of more powerful and more general tools. Nevertheless, a graphical method based on combination of the value set concept and the zero exclusion condition [7] is very universal and it is applicable for the wide range of uncertainty structures, including the very complicated ones.

According to [7], the value set at given frequency $\omega \in \square$ is:

$$
p(j \omega, Q)=\{p(j \omega, q): q \in Q\}
$$

Practical construction of the value sets can be done by substituting $s$ for $j \omega$, fixing $\omega \in \square$ and letting $q$ range over $Q$. The zero exclusion condition for Hurwitz stability of family of continuous-time polynomials (8) is defined [7]: Suppose invariant degree of polynomials in the family, pathwise connected uncertainty bounding set $Q$, continuous coefficient functions $\rho_{k}(q)$ for $k=0,1,2, \ldots, n$ and at least one stable member $p\left(s, q^{0}\right)$. Then the family $P$ is robustly stable if and only if:

$$
0 \notin p(j \omega, Q) \quad \forall \omega \geq 0
$$

The more detailed information can be found in [7] and subsequently e.g. in [9]. 


\section{Illustrative example}

Consider a third order interval plant [5]:

$$
G\left(s, b_{i}, a_{i}\right)=\frac{b_{2} s^{2}+b_{1} s+b_{0}}{a_{3} s^{3}+a_{2} s^{2}+a_{1} s+a_{0}}=\frac{[0.7 ; 1.3] s^{2}+[0.7 ; 1.3] s+[0.7 ; 1.3]}{[0.7 ; 1.3] s^{3}+[0.7 ; 1.3] s^{2}+[0.7 ; 1.3] s+[0.7 ; 1.3]}
$$

The transfer function of the nominal system with fixed mean values of the uncertain parameters is:

$$
G_{N}(s)=\frac{s^{2}+s+1}{s^{3}+s^{2}+s+1}
$$

and so the Diophantine equation (1) has the form:

$$
\left(s^{3}+s^{2}+s+1\right) s\left(p_{2} s^{2}+p_{1} s+p_{0}\right)+\left(s^{2}+s+1\right)\left(t_{3} s^{3}+t_{2} s^{2}+t_{1} s+t_{0}\right)=(s+m)^{6}
$$

which means that the case with multiple real roots is supposed. These multiple roots are selected as -1 , i.e. $m=1$. Moreover, the weight coefficients from (4) are assumed $\gamma_{1}=\gamma_{2}=\gamma_{3}=0.8$ which leads to the TFC:

$$
\begin{aligned}
& C_{Q}(s)=\frac{s^{2}+1.8 s+1}{s^{2}} \\
& C_{R}(s)=\frac{4 s^{3}+7.2 s^{2}+4 s+1}{s^{3}}
\end{aligned}
$$

The corresponding family of closed-loop characteristic polynomials with parameters from (11) is:

$$
\begin{aligned}
& p_{C L}\left(s, b_{i}, a_{i}\right)=a_{3} s^{6}+\left(a_{2}+5 b_{2}\right) s^{5}+\left(a_{1}+9 b_{2}+5 b_{1}\right) s^{4}+\left(a_{0}+5 b_{2}+9 b_{1}+5 b_{0}\right) s^{3}+\cdots \\
& +\left(b_{2}+5 b_{1}+9 b_{0}\right) s^{2}+\left(b_{1}+5 b_{0}\right) s+b_{0}
\end{aligned}
$$

The Fig. 2 shows the value sets of the family (16) for the frequency range from 0 to 5 with the step 0.01 . The zoomed version of the same plot, which provides the closer look to the neighborhood of the complex plane origin, is depicted in Fig. 3. It can be clearly seen that the origin of the complex plane (zero point) is excluded from the value sets. Moreover, the family contains a stable member. Consequently, the family of closed-loop characteristic polynomials (16) is robustly stable.

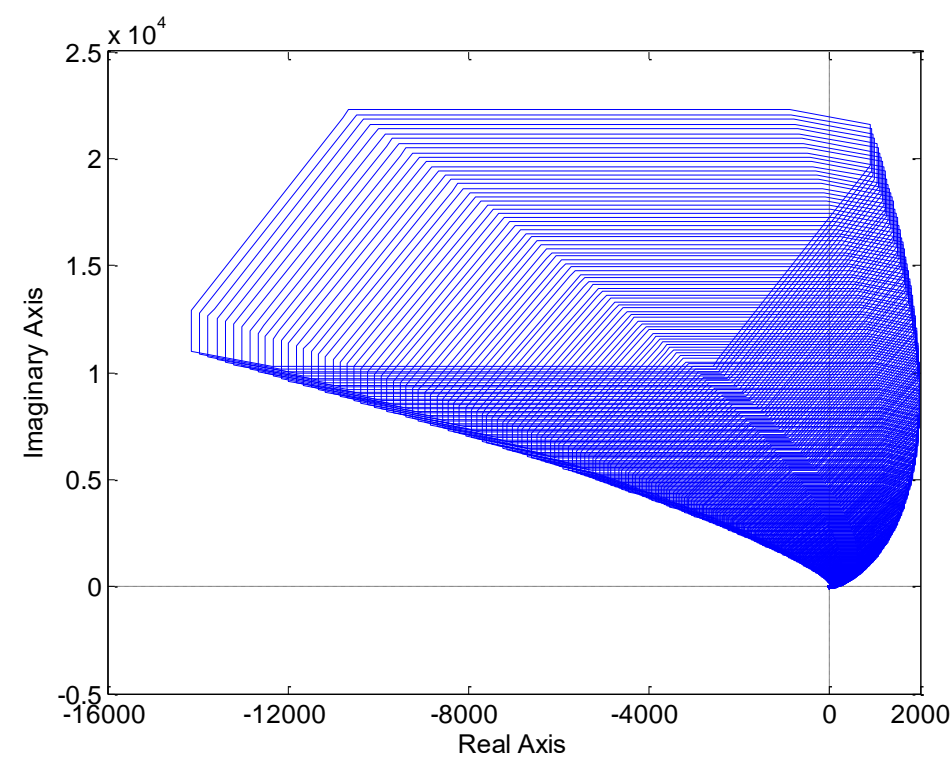

Fig. 2. Value sets for family of closed-loop characteristic polynomials (16) - full view 


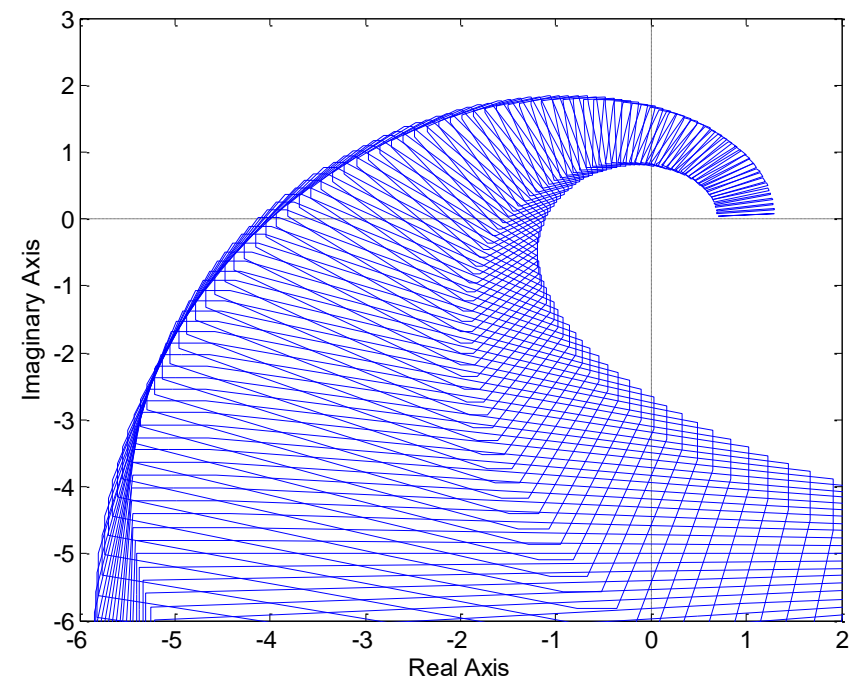

Fig. 3. Value sets for family of closed-loop characteristic polynomials (16) - zoomed view

The control simulations from Fig. 4 concur with the obtained result and also visually confirm robust stability of the designed circuit with TFC. More specifically, the Fig. 4 depicts the simulations of the output signals of 128 "sampled plants" from the interval family (11). For all seven interval parameters minimal and maximal values are taken and thus it leads to $2^{7}=128$ systems for simulation. Besides, the red curve represents the output signal of the nominal plant (12). Furthermore, it was assumed the stepwise reference signal changing from 1 to 2 in one third of the simulation time and the step load disturbance -1 affecting the input to the controlled plant during the last third of simulation.

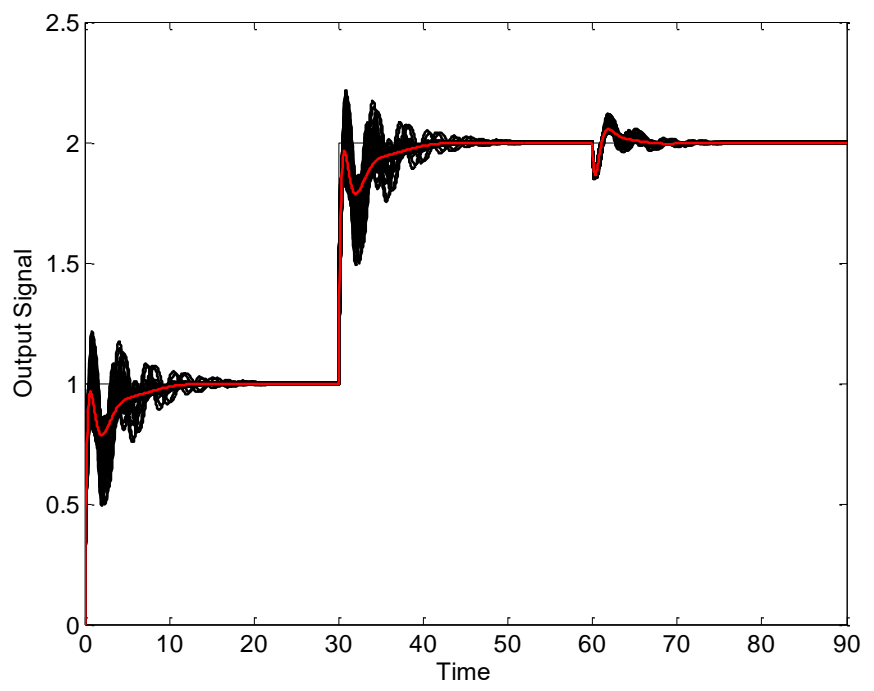

Fig. 4. Control of "sampled plants" from interval family (11) by TFC (14) and (15)

\section{Conclusion}

The contribution has been aimed to robust stability analysis of control loops with TFC and interval plants through visualization of the value sets and application of the zero exclusion condition. The TFC design itself is based on the polynomial method. In the illustrative example, a third order interval plant has been robustly stabilized by using TFC structure. The selection of weight coefficients would have no impact on the robust stability or instability of the control loop because the polynomial t(s) would remain the same. It could influence "only" the control performance.

\section{Acknowledgements}

This work was supported by the Ministry of Education, Youth and Sports of the Czech Republic within the National Sustainability Programme project No. LO1303 (MSMT-7778/2014). 


\section{References}

[1] P. Dostál, F. Gazdoš, V. Bobál, J. Vojtěšek, Adaptive control of a continuous stirred tank reactor by two feedback controllers, In: Proceedings of the 9th IFAC Workshop on Adaptation and Learning in Control and Signal Processing, Saint Petersburg, Russia, 2007.

[2] P. Dostál, F. Gazdoš, V. Bobál, Design of Controllers for Time Delay Systems: Integrating and Unstable Systems, In: Time-Delay Systems, InTech, Rijeka, Croatia, 2011, pp. 113-126.

[3] R. Matušů, R. Prokop, Robust stability of control systems with two feedback controllers and interval plants, In: Proceedings of the 19th International Conference on Systems, Zakynthos, Greece, 2015.

[4] R. Matušů, Robust stabilization of interval plants by means of two feedback controllers, International Journal of Circuits, Systems and Signal Processing, Vol. 9, 2015, pp. 427-434.

[5] R. Matušů, R. Prokop, Control of Interval Systems Using 2DOF Configuration, Procedia Engineering, Vol. 100 (25th DAAAM International Symposium on Intelligent Manufacturing and Automation, DAAAM 2014), 2015, pp. 340-344, ISSN 1877-7058.

[6] V. Kučera, Diophantine equations in control - A survey, Automatica, Vol. 29, No. 6 (1993), pp. 1361-1375.

[7] B. R. Barmish, New Tools for Robustness of Linear Systems, Macmillan, New York, USA, 1994.

[8] R. Ortega, R. Kelly, PID Self-Tuners: Some Theoretical and Practical Aspects, IEEE Transactions on Industrial Electronics, Vol. 31, No. 4 (1984), pp. 332-338.

[9] R. Matušů, R. Prokop, Graphical analysis of robust stability for systems with parametric uncertainty: an overview, Transactions of the Institute of Measurement and Control, Vol. 33, No. 2 (2011), pp. 274-290. 